\title{
A New CPG Model for the Generation of Modular Trajectories for Hexapod Robots
}

\author{
Carla M.A. Pinto, Diana Rocha* and Cristina P. Santos, Vítor Matos ${ }^{\dagger}$ \\ * Instituto Superior de Engenharia do Porto \\ and Centro de Matemática da Universidade do Porto \\ Rua Dr António Bernardino de Almeida, 431, \\ 4200-072 Porto, Portugal \\ ${ }^{\dagger}$ Universidade do Minho \\ Dept. Electrónica Industrial \\ Campus de Azurém \\ 4800-058 Guimarães \\ Portugal
}

\begin{abstract}
Legged robots are often used in a large variety of tasks, in different environments. Nevertheless, due to the large number of degrees-of-freedom to be controlled, online generation of trajectories in these robots is very complex. In this paper, we consider a modular approach to online generation of trajectories, based on biological concepts, namely Central Pattern Generators (CPGs). We introduce a new CPG model for hexapod robots' rhythms, based in the work of Golubitsky et al (1998). Each neuron/oscillator in the CPG consists of two modules/primitives: rhythmic and discrete. We study the effect on the robots' gaits of superimposing the two motor primitives, considering two distinct types of coupling. We conclude, from the simulation results, that the amplitude and frequency of periodic solutions, identified with hexapods' tripod and metachronal gaits, remain constant for the two couplings, after insertion of the discrete part.
\end{abstract}

Keywords: stability, CPG, modular locomotion, rhythmic primitive, discrete primitive

\section{INTRODUCTION}

In the last few years, there has been a large development in the modeling of online generation of trajectories in legged robots. Models producing rhythmic robots' patterns, inspired in biology, are now common. Locomotion in vertebrates is commonly structured in three layers [8], the top are the brainstem command systems, the structures that decide which motor pattern is to be activated at each moment of time. The middle layer consists of the steering and posture control systems. The bottom layer includes the Central Pattern Generators (CPGs). CPGs are networks of neurons located at the spinal level of vertebrates responsible for the rhythmic patterns observed during animals' locomotion. Mathematically, CPGs are commonly modeled by coupled nonlinear dynamical systems [6, 7, 4]. Dynamical systems have nice properties, such as smooth modulation, low computational cost, phase-locking between oscillators, extremely useful to online modulation of trajectories [10, 3]. Matos et al [10] propose a bio-inspired robotic controller able to generate locomotion and to easily switch between different types of gaits. Campos et al [3], present a two-layer architecture to model hexapod robots' locomotion. The bottom layer consists of the CPG for generating hexapods' gaits and the second layer sets up the parameter values for each gait. They study smooth gait transition in the model, using a modulatory drive signal regulating CPG's activity. Authors also propose a lateral posture control, based on dynamical systems, that corrects the robot posture and keeps its balance, when subject to changes in the lateral tilt. In this paper, we study the CPG model hexapod-robot for modular generation of an hexapod robot movements, using a biological approach [1]. CPG hexapod-robot is a network of twelve coupled CPG-units, each of which consists of two motor primitives: rhythmic and discrete. We study the variation in the amplitude and the frequency values of the periodic solutions produced by the CPG model hexapod-robot, and identified with common hexapods' gaits. We consider two types of couplings between the CPG units, diffusive and synaptic. The main goal is to show that these discrete corrections may be performed since that they do not affect the gait, meaning that the amplitude and frequency of the resultant trajectories is kept constant. Amplitude and frequency may be identified, respectively, with the range of motion and the velocity of the robot's movements, when considering implementations of the proposed controllers for generating trajectories for the joints of real robots.

Numerical Analysis and Applied Mathematics ICNAAM 2011

AIP Conf. Proc. 1389, 504-508 (2011); doi: 10.1063/1.3636775

(C) 2011 American Institute of Physics 978-0-7354-0956-9/\$30.00 


\section{CPG MODEL FOR HEXAPODS}

In this section we introduce a CPG model for online generation of trajectories of hexapod robots. It is based in the work of Golubitsky et al [7]. We give the general class of systems of ODEs that model CPG hexapod-robot and resume the symmetry techniques that allow classification of periodic solutions produced by this CPG model and identified with common hexapod locomotor rhythms.

Figure 1 shows the CPG model hexapod-robot(Fig 1) for generating locomotion for hexapods robots. It consists of twelve coupled oscillators. The oscillators (or cells) are denoted by circles and the arrows represent the couplings between cells. Each cell is a CPG unit and is divided onto two motor primitives, discrete and rhythmic, modeled by simple nonlinear dynamical systems. There are two types of couplings that force the network to have

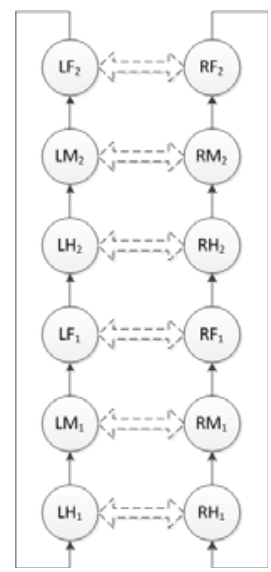

FIGURE 1. CPG locomotor model for hexapods, hexapod-robot. LF (left fore leg), LM (left middle leg), LH (left hind leg), RF (right fore leg), RM (right middle leg), RH (right hind leg).

$$
\Gamma_{\text {hexapod-robot }}=\mathbf{Z}_{6}(\omega) \times \mathbf{Z}_{2}(\kappa)
$$

symmetry. The CPG model hexapod-robot has the bilateral symmetry of animals $\left(\mathbf{Z}_{2}(\kappa)\right)$ and a translational symmetry $\left(\mathbf{Z}_{6}(\omega)\right)$, from back to front, i.e, RF is coupled to cell $\mathrm{RH}$, and the same applies for cells on the left side.

The class of systems of differential equations of the CPG model hexapod-robot is of the form:

$$
\begin{aligned}
\dot{x}_{L H_{1}} & =F\left(x_{L H_{1}}, x_{R H_{1}}, x_{L F_{2}}\right) \\
\dot{x}_{R H_{1}} & =F\left(x_{R H_{1}}, x_{L H_{1}}, x_{R F_{2}}\right) \\
\dot{x}_{L M_{1}} & =F\left(x_{L M_{1}}, x_{R M_{1}}, x_{L H_{1}}\right) \\
\dot{x}_{R M_{1}} & =F\left(x_{R M_{1}}, x_{L M_{1}}, x_{R H_{1}}\right) \\
\dot{x}_{L F_{1}} & =F\left(x_{L F_{1}}, x_{R F_{1}}, x_{L M_{1}}\right) \\
\dot{x}_{R F_{1}} & =F\left(x_{R F_{1}}, x_{L F_{1}}, x_{R M_{1}}\right) \\
\dot{x}_{L H_{2}} & =F\left(x_{L H_{2}}, x_{R H_{2}}, x_{L F_{1}}\right) \\
\dot{x}_{R H_{2}} & =F\left(x_{R H_{2}}, x_{L H_{2}}, x_{R F_{1}}\right) \\
\dot{x}_{L M_{2}} & =F\left(x_{L M_{2}}, x_{R M_{2}}, x_{L H_{2}}\right) \\
\dot{x}_{R M_{2}} & =F\left(x_{R M_{2}}, x_{L M_{2}}, x_{R H_{2}}\right) \\
\dot{x}_{L F_{2}} & =F\left(x_{L F_{2}}, x_{R F_{2}}, x_{L M_{2}}\right) \\
\dot{x}_{R F_{2}} & =F\left(x_{R F_{2}}, x_{L F_{2}}, x_{R M_{2}}\right)
\end{aligned}
$$

where $x_{i} \in \mathbf{R}^{k}$ is the cell $i$ variables, $k$ is the dimension of the internal dynamics for each cell, and $F:\left(\mathbf{R}^{k}\right)^{3} \rightarrow \mathbf{R}^{k}$ is an arbitrary mapping, all cells/neurons are identical.

The Theorem $H / K[6]$ allows the identification of symmetry types of periodic solutions, produced by a given coupled cell network. These periodic solutions are then identified with animals locomotor rhythms. Let $x(t)$ be a periodic solution of an ODE $\dot{x}=f(x)$, with period normalized to 1 , and with symmetry group $\Gamma$. Let $H$ and $K$ be subgroups of $\Gamma$. Symmetries $K$ fix the solution pointwise, i.e., let $\gamma \in \Gamma$, then $\gamma x(t)=x(t)$. They are called spatial symmetries. On the other hand, $H$ fixes the solution setwise, i.e., $\gamma x(t)=x(t-\theta) \leftrightarrow x(t+\theta)=x(t)$, where $\theta$ is the phase shift 
associated to $\gamma . H$ is the subgroup of spatio-temporal symmetries of the solution. If $\theta=0$, then $\gamma$ is a spatial symmetry. In order for $(H, K)$ to correspond to symmetries of a periodic solution $x(t)$ to (1) for some function $F$ the quotient $H / K$ must be cyclic. As an example, we present four of those pairs of symmetry types $(H, K)$ such as $H / K$ is cyclic. In Table 1, we show those pairs and their identification with common hexapod rhythms, pronk, lurch, metachronal, and tripod. Table 2, exhibits the corresponding periodic solutions. We briefly explain the identification of the hexapod tri-

TABLE 1. Symmetry pairs of periodic solutions, produced by the coupled cells system (1), and corresponding gaits.

\begin{tabular}{|l|l|l|}
\hline \multicolumn{1}{|c|}{$H$} & \multicolumn{1}{c|}{$K$} & Gait \\
\hline$\Gamma_{\text {hexapod-robot }}$ & $\Gamma_{\text {hexapod-robot }}$ & pronk \\
\hline$\Gamma_{\text {hexapod-robot }}$ & $\mathbf{Z}_{2}(\omega \kappa)$ & tripod \\
\hline$\Gamma_{\text {hexapod-robot }}$ & $\mathbf{Z}_{2}\left(\omega^{2}\right)$ & lurch \\
\hline $\mathbf{Z}_{2}(\omega \kappa)$ & $\mathbf{1}$ & metach. \\
\hline
\end{tabular}

TABLE 2. Periodic solutions of system (1), identified with hexapods gaits, where period of solutions is normalized to 1 . We only show the first six cells, the others can be easily computed. $S$ is half period out of phase.

\begin{tabular}{|c|c|c|c|}
\hline Left & Middle & Right & Gait \\
\hline$\left(x_{L H}, x_{L H}\right)$ & $\left(x_{L H}, x_{L H}\right)$ & $\left(x_{L H}, x_{L H}\right)$ & pronk \\
\hline$\left(x_{L H}, x_{L H}^{S}\right)$ & $\left(x_{L H}^{S}, x_{L H}\right)$ & $\left(x_{L H}, x_{L H}^{S}\right)$ & tripod \\
\hline$\left(x_{L H}, x_{L H}\right)$ & $\left(x_{L H}^{S}, x_{L H}^{S}\right)$ & $\left(x_{L H}, x_{L H}\right)$ & lurch \\
\hline$\left(x_{L H}, x_{L H}^{S}\right)$ & $\left(x_{L H}^{S / 3}, x_{L H}^{4 S / 3}\right)$ & $\left(x_{L H}^{2 S / 3}, x_{L H}^{5 S / 3}\right)$ & metac. \\
\hline
\end{tabular}

pod to a periodic solution of CPG hexapod-robot with symmetry pairs $(H, K)=\left(\Gamma_{\text {hexapod-robot }}, \mathbf{Z}_{2}(\omega \kappa)\right)$. Consider $x(t)=\left(x_{1}(t), \ldots, x_{1} 2(t)\right)$ be a periodic solution produced by CPG model hexapod-robot. In order to understand transformation $\omega \kappa$, we first apply $\kappa$ to $x(t)$, obtaining $\hat{x}(t)=\left(x_{2}(t), x_{1}(t), x_{4}(t), x_{3}(t), x_{6}(t), x_{5}(t), x_{8}(t), x_{7}(t), x_{10}(t), x_{9}(t), x_{12}(t), x_{11}(t)\right)$. After, we apply $\omega$ to $\hat{x}(t)$, resulting in solution $\tilde{x}(t)=\left(x_{4}(t), x_{3}(t), x_{6}(t), x_{5}(t), x_{8}(t), x_{7}(t),\right)\left(x_{10}(t), x_{9}(t), x_{12}(t), x_{11}(t), x_{2}(t), x_{1}(t)\right)$. Thus, spatial symmetry $\omega \kappa$ forces the final solution to have the form $\bar{x}(t)=\left(x_{1}(t), x_{2}(t), x_{2}(t), x_{1}(t), x_{1}(t), x_{2}(t),\right)\left(x_{2}(t), x_{1}(t), x_{1}(t), x_{2}(t), x_{2}(t), x_{1}(t)\right)$ Applying $\omega \kappa$ to the tripod does not change that gait since the groups of cells $(1,4,5,8,9,12)$ and $(2,3,6,7,10,11)$ receive the same set of signals. Spatio-temporal symmetries $\Gamma_{\text {hexapod-robot force signals sent to the to groups of cells above to be equal }}$ and to be half period out of phase.

\section{NUMERICAL SIMULATIONS}

We simulate the CPG model hexapod-robot. In each CPG-unit, we consider two distinct approaches to superimpose discrete and rhythmic primitives. The discrete part $y(t)$ is inserted as an offset of the rhythmic part $x(t)$. The resulting system bifurcates between a unique point attractor and a limit cycle according to one single parameter, $\mu$ (see below). It is believed that this design enables to produce more complex movements modeled as periodic movements around time varying offsets. We also consider two distinct couplings between the oscillators: diffusive and synaptic. We start from a stable periodic solution, purely rhythmic. Then, we vary parameter $T$ in steps of 0.1 in the interval $[-25,25]$. For each value of $T$ we simulate until a stable periodic solution is obtained and then compute its amplitude and frequency values. Then, we restart the simulations for a new value of $T$. Numerical results are illustrated.

The system of ordinary differential equations that models the discrete primitive is the VITE model given by [2]:

$$
\begin{aligned}
\dot{v} & =\delta(T-p-v) \\
\dot{p} & =G \max (0, v)
\end{aligned}
$$

This set of differential equations generates a trajectory converging to the target position $T$, at a speed determined by the difference vector $T-p$, where $p$ models the muscle length, and $G$ is the go command. $\delta$ is a constant controlling 
the rate of convergence of the auxiliary variable $v$. This discrete primitive controls a synergy of muscles so that the limb moves to a desired end state, given a volitional target position.

The equations for the rhythmic motor primitive are known as modified Hopf oscillators $[9,10,3]$ and are given by:

$$
\begin{aligned}
& \dot{x}=\alpha\left(\mu-r^{2}\right) x-\omega z=f(x, z) \\
& \dot{z}=\alpha\left(\mu-r^{2}\right) z+\omega x=g(x, z)
\end{aligned}
$$

where $r^{2}=x^{2}+z^{2}, \sqrt{\mu}$ is the amplitude of the oscillation. For $\mu<0$ the oscillator is at a stationary state, and for $\mu>0$ the oscillator is at a limit cycle. At $\mu=0$ it occurs a Hopf bifurcation. Parameter $\omega$ is the intrinsic frequency of the oscillator, $\alpha$ controls the speed of convergence to the limit cycle. $\omega_{\text {swing }}$ and $\omega_{\text {stance }}$ are the frequencies of the swing and stance phases, $\omega(z)=\frac{\omega_{\text {stance }}}{\exp (-a z)+1}+\frac{\omega_{\text {swing }}}{\exp (a z)+1}$ is the intrinsic frequency of the oscillator. With this ODE system, we can explicitly control the ascending and descending phases of the oscillations as well as their amplitudes, by just varying parameters $\omega_{\text {stance }}, \omega_{\text {swing }}$ and $\mu$.

The coupled systems of ODEs that model CPG hexapod-robot for synaptic and diffusive couplings are given by:

$$
\begin{aligned}
\dot{x}_{i}= & f_{2}\left(x_{i}, z_{i}\right) \\
\dot{z}_{i}= & g_{2}\left(x_{i}, z_{i}\right)+k_{1} h_{1}\left(z_{i+1}, z_{i}\right)+ \\
& +k_{2} h_{2}\left(z_{i+2}, z_{i}\right)+k_{3} h_{3}\left(z_{i+3}, z_{i}\right)
\end{aligned}
$$

where $f_{2}\left(x_{i}, z_{i}\right)=f_{1}\left(x_{i}, z_{i}, y_{i}\right), g_{2}\left(x_{i}, z_{i}\right)=g_{1}\left(x_{i}, z_{i}, y_{i}\right)$ and $r_{i}^{2}=\left(x_{i}-y_{i}\right)^{2}+z_{i}^{2}$, Indices are taken modulo 4. Function $h_{l}\left(z_{j}, z_{i}\right), l=1,2,3$, represents synaptic coupling when written in the form $h_{l}\left(z_{j}, z_{i}\right)=z_{j}, l=1,2,3$, and diffusive coupling when written as $h_{l}\left(z_{j}, z_{i}\right)=z_{j}-z_{i}, l=1,2,3$.

We simulate the CPG model (4). Parameter values used in the simulations are $\mu=10.0, \alpha=5, \omega_{\text {stance }}=6.2832$ $\operatorname{rads}^{-1}, \omega_{\text {swing }}=6.2832 \mathrm{rads}^{-1}, a=50.0, G=1.0, \delta=10.0$. Figure 2 shows amplitude and frequency values of the periodic solutions produced by CPG hexapod-robot and identified with the tripod hexapod rhythms. The values of $T$ not plotted in the graphs are those for which the solution, after insertion of the discrete part, goes to a stable equilibrium. Note that we obtain analogous graphs for the metachronal. By observation of the graphs, we conclude
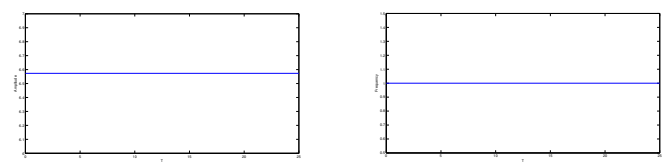

FIGURE 2. Amplitude (left) and frequency (right) of the periodic solutions produced by CPG hexapod-robot and identified with tripod, for varying $T \in[0,25]$ in steps of 0.1 , for diffusive and synaptic couplings.

that the amplitude and frequency values of the achieved stable periodic solutions, obtained after inserting the discrete to the rhythmic primitive, are not or are only slightly affected. Therefore, it is possible to use them for generating trajectories for the joint values of real robots, since varying the joint offset will not affect the required amplitude and frequency of the resultant trajectory, nor the gait.

\section{CONCLUSION}

We present a new CPG model for the locomotion rhythms of an hexapod robot, consisting of twelve CPG-units. We study the effect on two periodic solutions, produced by this CPG model hexapod-robot, identified with tripod and metachronal of superimposing discrete and rhythmic primitives. We simulate the CPG model for synaptic and diffusive couplings. We compute the amplitude and the frequency values of the stable periodic solutions, obtained after inserting the discrete part into the rhythmic one, for values of the discrete primitive target parameter $T \in[0,25]$. Numerical results show that amplitude and frequency values are almost constant for the two couplings.

\section{ACKNOWLEDGMENTS}

CP was supported by Research funded by the European Regional Development Fund through the programme COMPETE and by the Portuguese Government through the FCT - Fundação para a Ciência e a Tecnologia under the 
project PEst-C/MAT/UI0144/2011. This work was also funded by FEDER Funding supported by the Operational Program Competitive Factors COMPETE and National Funding supported by the FCT - Portuguese Science Foundation through project PTDC/EEACRO/100655/2008.

\section{REFERENCES}

1. E. Bizzi, A d'Avella, P Saltiel and M Trensch. Modular organization of spinal motor systems. The Neuroscientist 8 No 5 (2002) 437-442.

2. D. Bullock and S. Grossberg. The VITE model: a neural command circuit for generating arm and articulator trajectories. In J. Kelso, A. Mandell, and M. Shlesinger, editors, Dynamic patterns in complex systems, pp 206-305. (1988).

3. R. Campos, V. Matos, C.P. Santos. Hexapod Locomotion: a Nonlinear Dynamical Systems Approach. IECON 2010 - 36th Annual Conference on IEEE Industrial Electronics Society , (2010) 1546-1551 .

4. J.J. Collins and I. Stewart. Hexapodal gaits and coupled nonlinear oscillators. Biological Cybernetics (1993) 287-298.

5. S. Degallier, C.P. Santos, L. Righetti, and A. Ijspeert. Movement Generation using Dynamical Systems: A Drumming Hummanoid Robot. Humanoid's06 IEEE-RAS International Conference on Humanoid Robots. Genova, Italy (2006).

6. M. Golubitsky and I. Stewart. The symmetry perspective, Birkhauser, (2002).

7. M. Golubitsky, I. Stewart, P.-L. Buono, and J.J. Collins. A modular network for legged locomotion. Physica D 115 (1998) $56-72$.

8. S. Grillner, P. Wallén, K. Saitoh, A. Kozlov, B. Robertson. Neural bases of goal-directed locomotion in vertebrates - an overview. Brain Research Reviews 57 (2008) 2-12.

9. J. Marsden, and M. McCracken. Hopf Bifurcation and Its Applications. New York: Springer-Verlag, (1976).

10. V. Matos, C.P. Santos, C.M.A. Pinto. A Brainstem-like Modulation Approach for Gait Transition in a Quadruped Robot. Proceedings of The 2009 IEEE/RSJ International Conference on Intelligent RObots and Systems, IROS 2009 St Louis, MO, USA, October (2009). 